\title{
GLOBALIZATION OF SQP-METHODS IN CONTROL OF THE INSTATIONARY NAVIER-STOKES EQUATIONS
}

\author{
Michael HintermülleR ${ }^{1, *}$ And Michael Hinze ${ }^{2, \dagger}$
}

\begin{abstract}
A numerically inexpensive globalization strategy of sequential quadratic programming methods (SQP-methods) for control of the instationary Navier Stokes equations is investigated. Based on the proper functional analytic setting a convergence analysis for the globalized method is given. It is argued that the a priori formidable SQP-step can be decomposed into linear primal and linear adjoint systems, which is amenable for existing CFL-software. A report on a numerical test demonstrates the feasibility of the approach.
\end{abstract}

Mathematics Subject Classification. 49M05, 49M29, 49M37, 76D55.

Received: July 3, 2001. Revised: April 16, 2002.

\section{INTRODUCTION}

In this paper we are concerned with a numerically inexpensive globalization strategy of sequential quadratic programming methods (SQP-methods) in control of the instationary Navier Stokes equations. Thus the problem under consideration is

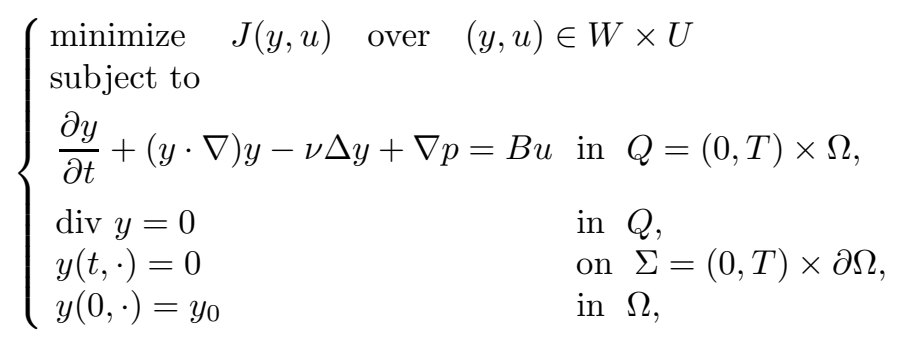

where $\Omega$ is a bounded domain in $\mathbb{R}^{2}$, and $T>0$ denotes the final time. In this form solving (1) appears at first to be a standard task. However, the formidable size of (1) and the goal of analyzing second order methods

\footnotetext{
Keywords and phrases. Globalized SQP-method, line search, Navier Stokes equations, optimal control.

1 Department of Mathematics, Karl-Franzens University of Graz, A-8010 Graz, Austria.

e-mail: michael.hintermueller@kfunigraz.ac.at

* The first author acknowledges support of the Austrian FWF under SFB 3 Optimization and Control.

2 Fakultät für Mathematik und Naturwissenschaften, TU-Dresden, D-01069 Dresden, Germany.

e-mail: hinze@math.tu-dresden.de

$\dagger$ The second author acknowledges support of the Special Research Grant SFB 557 Beeinflussung komplexer turbulenter Scherströmungen sponsored by the Deutsche Forschungsgemeinschaft. 
necessitate an independent analysis. One of the few contributions focusing on second order methods for optimal control of fluids are given by Ghattas et al. [5] and Heinkenschloss [9]. These works are restricted to stationary problems, however. Among other aspects analytical investigations on second order methods are given by the second author in [11] and by Kunisch and the second author in [12], where also further references can be found.

Traditionally, gradient-based methods were used in the design of solution algorithms in the control of the (instationary) Navier Stokes equations; see e.g. $[1,7]$. From a historical point of view, the analysis and application of second order methods is a rather recent development. One of the main advantages of second order methods consists in a very fast convergence behavior near a solution of (1) which is - of course - a desirable property of a numerical algorithm. In general, SQP-methods are only locally convergent which necessitates a sufficiently good initial guess for obtaining a convergent algorithm.

The main focus of the present paper is the introduction and analysis of a numerically inexpensive globalization strategy of SQP-methods in the control of the instationary Navier-Stokes equations, i.e. a method guaranteeing convergence of the SQP-algorithm independently of the initial choice. One key ingredient is the requirement of positive definiteness properties of the Hessian of the Lagrangian functional associated with (1). Under suitable convexity assumptions on $J$ the only term that might spoil the positive definiteness comes from the nonlinearity in the Navier Stokes equations. Exploiting the structure of the nonlinearity, we introduce a controlling parameter which is adjusted automatically in such a way that the appropriate positive definiteness properties of the Hessian of the Lagrangian are guaranteed.

From an optimization point of view the positive definiteness on the tangent space of the linearized constraints is required anyway for a well-defined SQP-method and is essential for our globalization strategy. Here the linearization is taken at an actual iterate of the algorithm. After solving an auxiliary quadratic optimization problem - the QP-part in SQP-methods - we use a line search in order to compute a suitable step-size along the actual direction. Again, we exploit the structure and can, therefore, obtain an explicit expression for an appropriate step size. This releases us from performing an expensive backtracking or interpolation strategy. The introduction of a step-size can also be interpreted as a damping strategy of Newton's method.

In the remainder of this section we introduce the appropriate functional analytic setting and discuss some known results on a priori estimates and differentiability of the state system in (1) in suitable spaces. In Section 2 we discuss first order and second order sufficient optimality conditions for (1). The globalization strategy is introduced in Section 3. First we consider the modification for obtaining Hessians fulfilling certain positive definiteness properties needed for SQP as well as for the second part of the globalization strategy. The latter one is achieved by a line search which is discussed next. In Section 4 global and local convergence results are proved. Finally, we report on numerical results attained by the proposed algorithm. This is essentially the contents of Section 5 .

To define the spaces and operators required for the investigation of (1) we introduce the solenoidal spaces

$$
H=\left\{v \in C_{0}^{\infty}(\Omega)^{2}: \operatorname{div} v=0\right\}^{-|\cdot|} L^{2}, V=\left\{v \in C_{0}^{\infty}(\Omega)^{2}: \operatorname{div} v=0\right\}^{-\mid \cdot H_{H}},
$$

with the superscripts denoting closures in the respective norms. Further we define

$$
W_{q}^{p}=\left\{v \in L^{p}(V): v_{t} \in L^{q}\left(V^{*}\right)\right\} \quad \text { and } \quad Z:=L^{2}(V) \times H,
$$

where $W_{q}^{p}$ is endowed with the norm

$$
|v|_{W_{q}^{p}}=|v|_{L^{p}(V)}+\left|v_{t}\right|_{L^{q}\left(V^{*}\right)}
$$

abbreviate $W:=W_{2}^{2}$ and set $\langle\cdot, \cdot\rangle:=\langle\cdot, \cdot\rangle_{L^{2}\left(V^{*}\right), L^{2}(V)}$, with $V^{*}$ denoting the dual space of $V$. Here $L^{2}(V)$ is an abbreviation for $L^{2}(0, T ; V)$ and similarly $L^{2}\left(V^{*}\right)=L^{2}\left(0, T ; V^{*}\right)$. Recall that up to a set of measure zero in $(0, T)$ elements $v \in W$ can be identified with elements in $C([0, T] ; H)$. In (1) further $U$ denotes the Hilbert space of controls which is identified with its dual $U^{*}$. We assume that the cost functional $J: W \times U \rightarrow \mathbb{R}$, $J(y, u)=J_{1}(y)+J_{2}(u)$, is bounded from below, weakly lower semi-continuous, twice Fréchet differentiable with 
locally Lipschitzian second derivative, and radially unbounded in $u$, i.e. $J(y, u) \rightarrow \infty$ as $|u|_{U} \rightarrow \infty$, for every $y \in W$.

Example 1.1. These assumptions are satisfied for cost functionals including tracking type functionals (also with tracking of states at the final time $T$ )

$$
J(y, u)=\frac{1}{2} \int_{Q}|y-z|^{2} \mathrm{~d} x \mathrm{~d} t+\frac{\gamma}{2} \int_{\Omega}|y(T)-z(T)|^{2} \mathrm{~d} x+\frac{\alpha}{2}|u|_{U}^{2},
$$

and functionals involving the vorticity of the fluid

$$
J(y, u)=\frac{1}{2} \int_{Q}\left|\nabla_{x} \times y(t, \cdot)\right|^{2} \mathrm{~d} x \mathrm{~d} t+\frac{\alpha}{2}|u|_{U}^{2},
$$

where $\alpha, \gamma>0$ and $z \in W$ are given. Of course, these functionals are even infinitely Fréchet differentiable on $W \times U$. Moreover, both functionals satisfy the positive definiteness Assumption (A1) on p. 732 and the structural Assumption (A2) on p. 736, respectively.

We define the nonlinear mapping

$$
e: W \times U \rightarrow Z^{*}
$$

by

$$
e(y, u)=\left(\frac{\partial y}{\partial t}+(y \cdot \nabla) y-\nu \Delta y-B u, y(0)-y_{0}\right)
$$

where $B \in \mathcal{L}\left(U, L^{2}\left(V^{*}\right)\right)$ denotes the control extension operator and $y_{0} \in H$. In variational form the constraints in (1) can be equivalently expressed as: given $u \in U$ find $y \in W$ such that $y(0)=y_{0}$ in $H$ and

$$
\left\langle y_{t}, v\right\rangle+\langle(y \cdot \nabla) y, v\rangle+\nu(\nabla y, \nabla v)_{L^{2}\left(L^{2}\right)}=\langle B u, v\rangle \quad \forall v \in L^{2}(V),
$$

and the control problem (1) can be rewritten as

$$
\min _{(y, u) \in W \times U} J(y, u) \text { subject to } e(y, u)=0 \text { in } Z^{*} .
$$

It is well known, see Temam [20], that for every $u \in U(4)$ admits a unique solution $y(u) \in W$. Therefore, with respect to existence (5) can equivalently be rewritten as

$$
\min \hat{J}(u)=J(y(u), u) \text { subject to } u \in U
$$

where $y(u) \in W$ satisfies $e(y(u), u)=0$. It is proved by Abergel et al. [1] that $(6)$ admits a solution $\left(y^{*}, u^{*}\right) \in$ $W \times U=: X$. The Lagrangian $L: X \times Z \rightarrow \mathbb{R}$ is given by

$$
L(x, \lambda)=J(x)+\langle e(x), \lambda\rangle_{Z^{*}, Z},
$$

and we anticipate that the SQP-method can be interpreted as Newton's algorithm applied to the equation

$$
L^{\prime}(x, \lambda)=0
$$

with $L^{\prime}(x, \lambda)$ denoting the gradient of the Lagrangian (7). 
We shall frequently refer to the variational solution of the linearized Navier-Stokes system and the adjoint equations in the solenoidal setting. For this purpose we state the following proposition which is proved in [11], compare also [12] for a similar analytic framework. It is essential for the analysis of SQP, Newton and quasiNewton methods.

Proposition 1.2. Let $y \in W, v_{0} \in H$ and $g \in L^{2}\left(V^{*}\right)$. Then the system of linearized Navier-Stokes equations

$$
A(y) v=\left(g, v_{0}\right) \text { in } Z^{*} \Leftrightarrow\left\{\begin{array}{l}
v_{t}+(v \cdot \nabla) y+(y \cdot \nabla) v-\nu \Delta v=g \quad \text { in } L^{2}\left(V^{*}\right) \\
v(0)=v_{0} \text { in } H,
\end{array}\right.
$$

admits a unique variational solution $v \in W$. For every $f \in W^{*}$ the adjoint equation

$$
A(y)^{*} w=f \quad \text { in } W^{*}
$$

admits a unique variational solution $w=\left(w^{1}, w^{0}\right) \in Z$. If $f \in L^{q}\left(V^{*}\right) \cap W^{*}(1 \leq q \leq \infty)$, then for every $0 \leq \epsilon \leq \min \left\{q-1, \frac{1}{3}\right\}$ the function $w^{1}$ is an element of $W_{1+\epsilon}^{2}$ and the variational solution of

$$
\left\{\begin{array}{l}
-w_{t}^{1}+(\nabla y)^{t} w^{1}-(y \cdot \nabla) w^{1}-\nu \Delta w^{1}=f \\
w^{1}(T)=0
\end{array}\right.
$$

and it satisfies $w^{1}(0)=w^{0}$. The functions $v$ and $w^{1}$ satisfy the a priori estimates

(i) $|v|_{L^{\infty}(H)}+|v|_{L^{2}(V)} \leq C\left(|y|_{L^{2}(V)}\right)\left\{|g|_{L^{2}\left(V^{*}\right)}+\left|v_{0}\right|_{H}\right\}$.

(ii) $\left|v_{t}\right|_{L^{2}\left(V^{*}\right)} \leq C\left(|y|_{L^{2}(V)},|y|_{L^{\infty}(H)}\right)\left\{|g|_{L^{2}\left(V^{*}\right)}+\left|v_{0}\right|_{H}\right\}$.

(iii) $\left|w^{1}\right|_{L^{2}(V)} \leq C\left(|y|_{L^{2}(V)},|y|_{L^{\infty}(H)}\right)|f|_{W^{*}}$.

(iv) $\left|w_{t}^{1}\right|_{L^{1+\epsilon}\left(V^{*}\right)} \leq C\left(T^{\frac{1-\epsilon}{2(1+\epsilon)}}, T^{\frac{1-3 \epsilon}{4(1+\epsilon)}},|y|_{L^{2}(V)},|y|_{L^{\infty}(H)}\right)$ $\times\left\{|f|_{W^{*}}+|f|_{L^{1+\epsilon}\left(V^{*}\right)}\right\}$ for all $\left(0 \leq \epsilon \leq \min \left\{q-1, \frac{1}{3}\right\}\right)$.

If, in addition, $y \in L^{\infty}(V)$ and $f \in L^{2}\left(V^{*}\right)$, then $w^{1} \in W$ and

(v) $\left|w^{1}\right|_{L^{2}(V)}+\left|w_{t}^{1}\right|_{L^{2}\left(V^{*}\right)} \leq C\left(|y|_{L^{\infty}(V)}\right)|f|_{L^{2}\left(V^{*}\right)}$.

For $y \in W \cap L^{\infty}(V) \cap L^{2}\left(H^{2}(\Omega)^{2}\right), v_{0} \in V$ and $g, f \in L^{2}(H)$ the unique solutions $v$ of (8) and $w^{1}$ of (9) are elements of $H^{2,1}(Q)$ and satisfy the a priori estimates

(vi) $|v|_{H^{2,1}(Q)} \leq C\left(|y|_{L^{\infty}(V)},|y|_{L^{2}\left(H^{2}(\Omega)^{2}\right)}\right)\left\{|g|_{L^{2}(H)}+\left|v_{0}\right|_{V}\right\}$

and

(vii) $\left|w^{1}\right|_{H^{2,1}(Q)} \leq C\left(|y|_{L^{\infty}(V)},|y|_{L^{2}\left(H^{2}(\Omega)^{2}\right)}\right)|f|_{L^{2}(H)}$.

To apply the SQP-method to (1) we need second order information of the Lagrangian $L$. The basic ingredients are the derivatives of the operator $e$ which were characterized in [11], compare also [12]. For the convenience of the reader we state:

Proposition 1.3. The operator $e=\left(e^{1}, e^{2}\right): X \rightarrow Z^{*}$ is infinitely Fréchet differentiable with Lipschitz continuous first derivative, constant second derivative and vanishing third and higher derivatives. The action of the first two derivatives of $e^{1}$ are given by

$$
\left\langle e_{x}^{1}(x)(w, s), \phi\right\rangle=\left\langle w_{t}, \phi\right\rangle+\langle(w \cdot \nabla) y, \phi\rangle+\langle(y \cdot \nabla) w, \phi\rangle+\nu(\nabla w, \nabla \phi)_{L^{2}\left(L^{2}\right)}-\langle B s, \phi\rangle_{L^{2}\left(L^{2}\right)},
$$

where $x=(y, u) \in X,(w, s) \in X$ and $\phi \in L^{2}(V)$, and

$$
\begin{array}{r}
\left\langle e_{x x}^{1}(x)(w, s)(v, r), \phi\right\rangle=\left\langle e_{y y}^{1}(x)(w, v), \phi\right\rangle= \\
\langle(w \cdot \nabla) v, \phi\rangle+\langle(v \cdot \nabla) w, \phi\rangle=:\langle v, H(\phi) w\rangle_{W, W^{*}}
\end{array}
$$

where $(v, r) \in X$ and $H: L^{2}(V) \rightarrow \mathcal{L}\left(W, W^{*}\right)$. 
We close this section with recalling some basic properties of the trilinear form

$$
b(u, v, w):=\int_{\Omega}(u \cdot \nabla) v w \mathrm{~d} x
$$

which are proved in [20] (Chap. III). Note that the following estimates are the key essentials in the proofs of Propositions 1.2 and 1.3 .

Lemma 1.4. Let $\Omega \subset \mathbb{R}^{2}$ be a bounded domain, and denote by $S$ the Stokes operator. There holds $b(u, v, w)=$ $-b(u, w, v)$ for all $u, v, w \in V$ and

$$
b(u, v, w) \leq C \begin{cases}|u|_{H}^{\frac{1}{2}}|u|_{V}^{\frac{1}{2}}|v|_{H}^{\frac{1}{2}}|v|_{V}^{\frac{1}{2}}|w|_{V} & \forall u, v, w \in V \\ |u|_{H}^{\frac{1}{2}}|u|_{V}^{\frac{1}{2}}|v|_{V}^{\frac{1}{2}}|S v|_{H}^{\frac{1}{2}}|w|_{H} & \forall u \in V, v \in V \cap H^{2}(\Omega)^{2}, w \in H \\ |u|_{H}|v|_{V}|w|_{H}^{\frac{1}{2}}|S w|_{H}^{\frac{1}{2}} & \forall u \in H, v \in V, w \in V \cap H^{2}(\Omega)^{2} \\ |u|_{H}^{\frac{1}{2}}|S u|_{H}^{\frac{1}{2}}|v|_{V}|w|_{H} & \forall u \in V \cap H^{2}(\Omega)^{2}, v \in V, w \in H,\end{cases}
$$

with a positive constant $C$. The estimates are also valid for $H$ replaced by $L^{2}(\Omega)^{2}, V$ replaced by $H^{1}(\Omega)^{2}$ and $|S u|_{H}$ replaced by $|u|_{H^{2}(\Omega)^{2}}$.

Note that the first estimate in (12) immediately implies that $b(u, u, \cdot) \in L^{2}\left(V^{*}\right)$ for $y \in W$, see for example [20] (Chap. III).

\section{Optimality COnditions}

In this section we summarize first order necessary and second order sufficient optimality conditions for problem (1).

\subsection{First order necessary optimality condition}

A sufficient condition for the existence of Lagrange multipliers associated to solutions $x^{*}=\left(y^{*}, u^{*}\right)$ of the constrained minimization problem (1) is given by the surjectivity of the operator $e_{x}\left(x^{*}\right)$.

Theorem 2.1. Let $x \in X$. Then the operator $e_{x}(x): X \rightarrow Z^{*}$ is surjective.

Proof. Let $\left(f, v_{0}\right) \in Z^{*}$ and $\tilde{u} \in U$ arbitrary, but fixed. Due to Proposition 1.2 the equation

$$
e_{x}(x)(v, \tilde{u})=\left(f, v_{0}\right) \quad \text { in } Z^{*} \Longleftrightarrow e_{y}(x) v=\left(f+B \tilde{u}, v_{0}\right) \quad \text { in } Z^{*}
$$

admits a unique solution $v \in W$ with $v(0)=v_{0}$. Since $\tilde{u}$ was chosen arbitrarily, this proves the claim.

Theorem 2.2 (Existence and uniqueness of Lagrange multipliers). Let $x^{*}=\left(y^{*}, u^{*}\right) \in X$ be a solution of the optimization problem (1). Then there exists a unique Lagrange multiplier $\lambda^{*} \in Z$ which, together with the optimal solution $x^{*}$, satisfies the optimality system

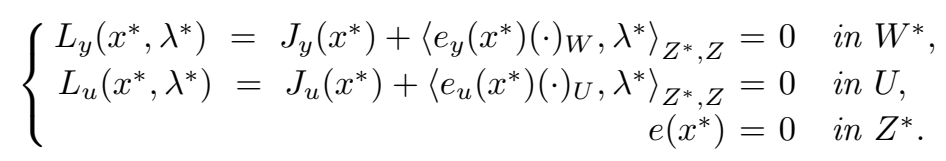

Furthermore, if $J_{y}\left(x^{*}\right) \in L^{q}\left(V^{*}\right) \cap W^{*}(1 \leq q \leq \infty)$ then there holds $\lambda^{*}=\left(\lambda^{*^{1}}, \lambda^{*^{0}}\right) \in W_{1+\epsilon}^{2} \times H$ for all 
$\left(0 \leq \epsilon \leq \min \left(q-1, \frac{1}{3}\right)\right)$ with $\lambda^{*^{0}}=\lambda^{*^{1}}(0)$ and $\lambda^{*^{1}}$ satisfies the a priori estimates

L1. $\left|\lambda^{*^{1}}\right|_{L^{2}(V)} \leq C\left(\left|y^{*}\right|_{L^{2}(V)},\left|y^{*}\right|_{L^{\infty}(H)}\right)\left|J_{y}\left(x^{*}\right)\right|_{W^{*}}$,

L2. $\left|\lambda_{t}^{*^{1}}\right|_{L^{1+\epsilon}\left(V^{*}\right)} \leq C\left(T^{\frac{1-\epsilon}{2(1+\epsilon)}}, T^{\frac{1-3 \epsilon}{4(1+\epsilon)}},\left|y^{*}\right|_{L^{2}(V)},\left|y^{*}\right|_{L^{\infty}(H)}\right)\left\{\left|J_{y}\left(x^{*}\right)\right|_{L^{1+\epsilon}\left(V^{*}\right)}\right.$

$$
\left.+\left|J_{y}\left(x^{*}\right)\right|_{W^{*}}\right\}\left(0 \leq \epsilon \leq \min \left(q-1, \frac{1}{3}\right)\right) .
$$

Proof. Since $e_{y}\left(x^{*}\right)=A(y)^{*}$ with $A(y)$ defined in (8) and $J_{y}\left(x^{*}\right) \in W^{*}$ the first part of the theorem follows from Proposition 1.2 with $w$ replaced by $\lambda$ and $f$ replaced by $J_{y}\left(x^{*}\right)$. The second part follows from the same proposition, (iii) and (iv), since now $J_{y}\left(x^{*}\right) \in L^{q}\left(V^{*}\right) \cap W^{*}$.

\subsection{Second order conditions}

In order to provide convergence analysis of the SQP-method it will be essential to derive conditions that ensure a (strong) second order sufficient optimality condition for the Lagrangian $L(x, \lambda)$. The key to these conditions are the a priori estimates of Proposition 1.2. In the first result we assert positive definiteness of the reduced Hessian associated with (1) provided that $J_{y}(x)$ is sufficiently small, a condition which is applicable to control problems of tracking-type, say.

Lemma 2.3 (Positive definiteness of Reduced Hessian). Let $u \in U$ and assume that $J_{y y}(x) \in \mathcal{L}\left(W, W^{*}\right)$ is positive semi-definite and $J_{u u}(x) \in \mathcal{L}(U)$ is positive definite, where $x=(y(u), u)$. Then, the reduced Hessian

$$
H(x, \lambda):=T^{*}(x) L_{x x}(x, \lambda) T(x)
$$

is positive definite provided that $\left|J_{y}(x)\right|_{W^{*}}$ is sufficiently small. Here,

$$
T(x): U \rightarrow X, \quad T(x):=\left[\begin{array}{c}
-e_{y}(x)^{-1} e_{u}(x) \\
I d_{U}
\end{array}\right] .
$$

Proof. Since

$$
H(x, \lambda)=e_{u}^{*}(x) e_{y}^{-*}(x) J_{y y}(x) e_{y}^{-1}(x) e_{u}(x) \cdot+e_{u}^{*}(x) e_{y}^{-*}(x)\left\langle e_{y y}^{1}(x)\left(e_{y}^{-1}(x) e_{u}(x) \cdot, \cdot\right), \lambda^{1}(x)\right\rangle+J_{u u}(x) \cdot
$$

one has

$$
(H(x, \lambda) s, s)_{U}=\left\langle J_{y y}(x) w, w\right\rangle_{W^{*}, W}+\left\langle H\left(\lambda^{1}\right) w, w\right\rangle_{W^{*}, W}+\left(J_{u u}(x) s, s\right)_{U},
$$

where the mapping $H$ is defined in (11) and $w:=-e_{y}^{-1}(x) e_{u}(x) s$. The third addend in this equation is bounded from below by a constant times $|u|_{U}^{2}$ and, since $J_{y y}(x)$ is positive semi-definite the first addend is non-negative. In order to tackle the second addend define

$$
\mathcal{R}:=e_{u}^{*}(x) e_{y}^{-*}(x)\left\langle e_{y y}^{1}(x)\left(e_{y}^{-1}(x) e_{u}(x) \cdot, \cdot\right), \lambda^{1}(x)\right\rangle \in \mathcal{L}(U)
$$

and recall that for $g, h \in W$

$$
\left\langle e_{y y}^{1}(x)(g, h), \lambda^{1}(x)\right\rangle=\int_{0}^{T} \int_{\Omega}(g \cdot \nabla) h \lambda^{1}+(h \cdot \nabla) g \lambda^{1} \mathrm{~d} x \mathrm{~d} t .
$$

Utilizing the continuity of the embedding $W \hookrightarrow L^{\infty}(H)$ and the definition of $w$ together with Proposition 1.2 one estimates

$$
\left|\left\langle e_{y y}^{1}(x)(w, w), \lambda^{1}(x)\right\rangle\right| \leq C|w|_{W}^{2}\left|\lambda^{1}\right|_{L^{2}(V)} \leq C|s|_{U}^{2}\left|J_{y}(x)\right|_{W^{*}}
$$


with a generic positive constant $C$ independent of $w$. Therefore,

$$
(\mathcal{R} s, s)_{U} \geq-C|s|_{U}^{2}\left|J_{y}(x)\right|_{W^{*}}
$$

Now let $\delta>0$ such that $\left(J_{u u}(x) s, s\right)_{U} \geq \delta|s|_{U}^{2}$. Then the above estimate for $\mathcal{R}$ implies

$$
\left(\hat{J}^{\prime \prime}(u) s, s\right)_{U} \geq\left(\delta-C\left|J_{y}(x)\right|_{W^{*}}\right)|s|_{U}^{2},
$$

which is the claim.

Lemma 2.4. Let $x \in X$ and denote by $\lambda=\lambda(x) \in Z$ the solution of $(9)$, with $f=-J_{y}(x)$, associated to $x$. Let $J_{y y}(x) \in \mathcal{L}\left(W, W^{*}\right)$ be positive semi-definite, let $J_{u u}(x) \in \mathcal{L}(U)$ be positive definite and let $\left|J_{y}(x)\right|_{W^{*}}$ be sufficiently small. Then, $L_{x x}(x, \lambda)$ is positive definite on the kernel of $e_{x}(x)$, i.e.

$$
\left\langle L_{x x}(x, \lambda) x, x\right\rangle_{X^{*} X} \geq c|x|_{X}^{2} \quad \text { for all } x \in \mathcal{N}\left(e_{x}(x)\right)
$$

with a positive constant $c$ independent of $x$.

Proof. Let $(v, \tilde{u}) \in \mathcal{N}\left(e_{x}(x)\right)$. Then $v$ solves (8) with $v_{0}=0$ and $g=B \tilde{u}$. Due to Proposition $1.2, v \in W$ and satisfies

$$
|v|_{W} \leq C\left(|y|_{L^{2}(V)},|y|_{L^{\infty}(H)},\|B\|_{\mathcal{L}\left(U, L^{2}\left(V^{*}\right)\right)}\right)|\tilde{u}|_{U}
$$

Let $\delta>0$ be chosen such that $J_{u u}(x)(\tilde{u}, \tilde{u}) \geq \delta|\tilde{u}|_{U}^{2}$. One finds

$$
\begin{aligned}
\left\langle L_{x x}(x, \lambda)(v, \tilde{u}),(v, \tilde{u})\right\rangle_{X^{*}, X} & =J_{y y}(x)(v, v)+\left\langle e_{y y}^{1}(x)(v, v), \lambda^{1}\right\rangle+J_{u u}(x)(\tilde{u}, \tilde{u}) \\
& \geq \delta|\tilde{u}|_{U}^{2}-2 \sqrt{2} \int_{0}^{T}|v|_{H}|v|_{V}\left|\lambda^{1}\right|_{V} \mathrm{~d} t \geq \delta|\tilde{u}|_{U}^{2}-C|\tilde{u}|_{U}^{2}\left|\lambda^{1}\right|_{L^{2}(V)} .
\end{aligned}
$$

As in the proof of Lemma 2.3

$$
\left\langle L_{x x}(x, \lambda)(v, \tilde{u}),(v, \tilde{u})\right\rangle_{X^{*}, X} \geq\left(\delta-C\left|J_{y}(x)\right|_{W^{*}}\right)|\tilde{u}|_{U}^{2},
$$

so that the claim follows.

\section{Globalization of the SQP-Method}

One way to motivate the classical SQP-approach is to consider the first order necessary conditions (13) of problem (5) and to apply Newton's method for its solution. Let $x^{*}$ be a local minimizer of (5) with corresponding optimal multiplier $\lambda^{*}$. Then the basic SQP-algorithm reads as follows [12]:

\section{Algorithm 3.1. SQP-algorithm}

1. Choose $\left(x^{0}, \lambda^{0}\right)$ sufficiently close to $\left(x^{*}, \lambda^{*}\right)$, and set $n=0$.

2. Do until convergence

(a) solve

$$
\left(\begin{array}{cc}
L_{x x}\left(x^{n}, \lambda^{n}\right) & e_{x}^{*}\left(x^{n}\right) \\
e_{x}\left(x^{n}\right) & 0
\end{array}\right)\left(\begin{array}{c}
\delta_{x}^{n} \\
\hat{\lambda}^{n}
\end{array}\right)=-\left(\begin{array}{c}
J_{x}\left(x^{n}\right) \\
e\left(x^{n}\right)
\end{array}\right)
$$

(b) update $x^{n+1}=x^{n}+\delta_{x}^{n}$ and $\lambda^{n+1}=\hat{\lambda}^{n}$, and set $n=n+1$. 
It is well known that under suitable assumptions (18) is the first order condition for

$$
\begin{aligned}
\text { minimize } & J\left(x^{n}\right)+J_{x}\left(x^{n}\right) \delta_{x}+\frac{1}{2} L_{x x}\left(x^{n}, \lambda^{n}\right)\left(\delta_{x}\right)^{2} \quad \text { over } \quad \delta_{x} \in X, \\
\text { subject to } & e\left(x^{n}\right)+e_{x}\left(x^{n}\right) \delta_{x}=0
\end{aligned}
$$

Here and below we use $J_{x}\left(x^{n}\right) \delta_{x}=\left\langle J_{x}\left(x^{n}\right), \delta_{x}\right\rangle_{X^{*}, X}, L_{x x}\left(x^{n}, \lambda\right)\left(\delta_{x}\right)^{2}=\left\langle L_{x x}\left(x^{n}, \lambda\right) \delta_{x}, \delta_{x}\right\rangle_{X^{*}, X}$, and analogously for $e_{x}, J_{x x}$. For the SQP-algorithm to be well defined one has to ensure positive definiteness of the Hessian $L_{x x}\left(x^{n}, \lambda^{n}\right)$ on the tangent space of the linearized constraints for all $n$; see [12] for details. Usually, this condition is satisfied only if the starting point $\left(x^{0}, \lambda^{0}\right)$ is in a sufficiently small neighborhood of a local minimizer satisfying the strong second order sufficient conditions (16). Even if $L_{x x}\left(x^{n}, \lambda^{n}\right)$ happens to fulfill the positive definiteness requirements for all $n$, no convergence may be achieved, since the full step along $\delta_{x}^{n}$ may not achieve a reasonable compromise between the two goals of minimizing $J$ and realizing $e(x)=0$.

Therefore our aim is twofold. First we analyze the structure of $L_{x x}(x, \lambda)$ in order to introduce a suitable modification whenever $L_{x x}\left(x^{n}, \lambda^{n}\right)$ is not (or not sufficiently) positive definite on the tangent space of the linearized constraints. To overcome the second drawback mentioned above, a line search is included. Again the problem structure is exploited to compute (in a numerically inexpensive way) a suitable step-length $\alpha^{n} \in(0,1]$ along the actual direction $\left(\delta_{x}^{n}, \delta_{\lambda}^{n}\right)$, with $\delta_{\lambda}^{n}=\hat{\lambda}^{n}-\lambda^{n}$, in order to obtain a descent behavior of an appropriate merit function being a measure for the reduction of $J$ and the violation of $e(x)=0$ at $x^{n}$.

\subsection{Positive definiteness}

From now onwards we invoke the following assumption

$$
J_{u u}(x)(\delta u)^{2} \geq c|\delta u|_{U}^{2} \forall \delta u \in U, \quad \text { and } \quad J_{y y}(x)(\delta y)^{2} \geq 0 \quad \forall \delta y \in W .
$$

Let us start with a detailed analysis of (18). First we rewrite the system in terms of derivatives w.r.t. $y$ and $u$, i.e.

$$
\left\{\begin{aligned}
J_{y y}\left(x^{n}\right) \delta_{y}^{n}+\left\langle e_{y y}\left(x^{n}\right) \delta_{y}^{n}, \lambda^{n}\right\rangle_{Z^{*}, Z}+e_{y}^{*}\left(x^{n}\right) \hat{\lambda}^{n} & =-J_{y}\left(x^{n}\right), \\
J_{u u}\left(x^{n}\right) \delta_{u}^{n}+e_{u}^{*}\left(x^{n}\right) \hat{\lambda}^{n} & =-J_{u}\left(x^{n}\right), \\
e\left(x^{n}\right)+e_{y}\left(x^{n}\right) \delta_{y}^{n}+e_{u}\left(x^{n}\right) \delta_{u}^{n} & =0 .
\end{aligned}\right.
$$

Recall that $e: X \rightarrow Z^{*}:=L^{2}\left(V^{*}\right) \times H$ is given by

$$
e(x)=\left(e^{1}(y, u), e^{2}(y, u)\right)=\left(\frac{\partial y}{\partial t}+(y \cdot \nabla) y-\nu \Delta y-B u, y(0)-y_{0}\right) .
$$

Due to the properties of $e^{2}$ we obviously have $e_{u}^{2}(y, u)=0$ and $e_{y y}^{2}(y, u)=0$. It follows from Proposition 1.3 that for all $w, v \in W$ and $q \in L^{2}(V)$

$$
\left\langle e_{y y}^{1}(x)(w, v), q\right\rangle=\langle(w \cdot \nabla) v, q\rangle+\langle(v \cdot \nabla) w, q\rangle .
$$

One further obtains for all $\delta_{x}=\left(\delta_{y}, \delta_{u}\right) \in X, q \in L^{2}(V)$

$$
\begin{aligned}
\left\langle e^{1}(x)+e_{x}^{1}(x) \delta_{x}, q\right\rangle & =\left\langle e^{1}\left(x+\delta_{x}\right), q\right\rangle-\left\langle\left(\delta_{y} \cdot \nabla\right) \delta_{y}, q\right\rangle \\
& =\left\langle e^{1}\left(x+\delta_{x}\right), q\right\rangle-\frac{1}{2}\left\langle e_{y y}^{1}(x)\left(\delta_{y}, \delta_{y}\right), q\right\rangle .
\end{aligned}
$$


This yields

$$
\left\langle L_{x x}(x, \lambda) \delta_{x}, \delta_{x}\right\rangle_{X^{*}, X}=\left\langle J_{x x}(x) \delta_{x}, \delta_{x}\right\rangle_{X^{*}, X}+2\left\langle\left(\delta_{y} \cdot \nabla\right) \delta_{y}, \lambda^{1}\right\rangle,
$$

where $\lambda^{1} \in L^{2}(V)$ denotes the first component of $\lambda=\left(\lambda^{1}, \lambda^{0}\right) \in Z$. Recall also that $e_{x x}(x)$ is independent of $x$.

The preceding results enable us to reduce the system (20). First we use the last equation and the invertibility of $e_{y}$ to obtain

$$
\delta_{y}^{n}=-e_{y}^{-1}\left(x^{n}\right)\left(e\left(x^{n}\right)+e_{u}\left(x^{n}\right) \delta_{u}^{n}\right)
$$

Using (25) in the first line of (20) we derive

$$
\hat{\lambda}^{n}=e_{y}^{-*}\left(x^{n}\right)\left(L_{y y}\left(x^{n}, \lambda^{n}\right) e_{y}^{-1}\left(x^{n}\right)\left(e\left(x^{n}\right)+e_{u}\left(x^{n}\right) \delta_{u}^{n}\right)-J_{y}\left(x^{n}\right)\right)
$$

where $e_{y}^{-*}$ denotes the inverse of the adjoint of $e_{y}$. Using (26) in the second line of (20) we finally arrive at

$$
H\left(x^{n}, \lambda^{n}\right) \delta_{u}^{n}=-T^{*}\left(x^{n}\right) J_{x}\left(x^{n}\right)-r_{0}^{n}\left(x^{n}\right) .
$$

Here

$$
r_{0}^{n}\left(x^{n}\right)=e_{u}^{*}\left(x^{n}\right) e_{y}^{-*}\left(x^{n}\right) L_{y y}\left(x^{n}, \lambda^{n}\right) e_{y}^{-1}\left(x^{n}\right) e\left(x^{n}\right)
$$

and $T(x)$ and $H(x, \lambda)$ are defined in Lemma 2.3. This result is interesting in several ways: (i) Solving (27) and then performing backward substitution, i.e. computing $\delta_{y}^{n}$ from (25) and then $\hat{\lambda}^{n}$ from (26), gives an efficient way for solving the system (20). This is especially substantiated by the fact that typically the control only acts on part of the domain $\Omega$, and thus only a small problem must be solved in (27). (ii) The second term on the right hand side in $(27)$, i.e. $r_{0}^{n}\left(x^{n}\right)$, vanishes whenever $x^{n}$ is feasible, i.e. it satisfies $e\left(x^{n}\right)=0$ implying $r_{0}^{n}\left(x^{n}\right)=0$. Then (27) corresponds to the system for Newton's method [12]. (iii) The operator on the left hand side is the reduced Hessian which easily can be modified to enforce positive definiteness.

The last aspect addressed above is considered next. For arbitrary starting points $\left(x^{0},(\lambda)^{0}\right)$ the positive definiteness of $L_{x x}\left(x^{n}, \lambda^{n}\right)$ on the tangent space of the linearized constraints may get lost. But this property is needed for a well defined SQP-method $[4,16]$. In order to tackle this situation we employ the following strategy: define

$$
L^{n}(\gamma)=\left(\begin{array}{cc}
J_{y y}\left(x^{n}\right)+\gamma\left\langle e_{y y}^{1}\left(x^{n}\right)(\cdot, \cdot), \lambda^{1^{n}}\right\rangle & 0 \\
0 & J_{u u}\left(x^{n}\right)
\end{array}\right)
$$

Below we shall also use $L_{11}^{n}(\gamma)=J_{y y}\left(x^{n}\right)+\gamma\left\langle e_{y y}^{1}\left(x^{n}\right)(\cdot, \cdot), \lambda^{1^{n}}\right\rangle$. Note that

$$
L^{n}(1)=L_{x x}\left(x^{n}, \lambda^{n}\right) \quad \text { and } \quad L^{n}(0)=\left(\begin{array}{cc}
J_{y y}\left(x^{n}\right) & 0 \\
0 & J_{u u}\left(x^{n}\right)
\end{array}\right) .
$$

It follows from assumption (A1) that for $\delta_{x}=\left(\delta_{y}, \delta_{u}\right) \in X$ with $\left|\delta_{u}\right|_{U} \neq 0$

$$
L^{n}(0)\left(\delta_{x}\right)^{2} \geq c\left|\delta_{u}\right|_{U}^{2}>0
$$

holds.

Instead of (18) we consider now the system

$$
\left(\begin{array}{cc}
L^{n}(\gamma) & e_{x}^{*}\left(x^{n}\right) \\
e_{x}\left(x^{n}\right) & 0
\end{array}\right)\left(\begin{array}{c}
\tilde{\delta}_{x}^{n} \\
\tilde{\lambda}^{n}
\end{array}\right)=-\left(\begin{array}{c}
J_{x}\left(x^{n}\right) \\
e\left(x^{n}\right)
\end{array}\right)
$$


Letting $H^{n}(\gamma):=T^{*}\left(x^{n}\right) L^{n}(\gamma) T\left(x^{n}\right)$ the analogues of (26) and (27) become

$$
\tilde{\lambda}^{n}=e_{y}^{-*}\left(x^{n}\right)\left(L_{11}^{n}(\gamma) e_{y}^{-1}\left(x^{n}\right)\left(e\left(x^{n}\right)+e_{u}\left(x^{n}\right) \delta_{u}^{n}\right)-J_{y}\left(x^{n}\right)\right),
$$

and

$$
H^{n}(\gamma) \tilde{\delta}_{u}^{n}=-T^{*}\left(x^{n}\right) J_{x}\left(x^{n}\right)-r_{e}^{n}\left(x^{n} ; \gamma\right)
$$

where

$$
r_{e}^{n}\left(x^{n} ; \gamma\right)=e_{u}^{*}\left(x^{n}\right) e_{y}^{-*}\left(x^{n}\right) L_{11}^{n}(\gamma) e_{y}^{-1}\left(x^{n}\right) e\left(x^{n}\right) .
$$

Note that $r_{e}^{n}\left(x^{n}, 1\right)=r_{0}^{n}\left(x^{n}\right)$.

Next fix $\epsilon \in(0, c)$. Then step (2a) of the SQP-algorithm is replaced by:

\section{Step (2a)}

(i) $\operatorname{Set} \gamma^{n}:=1$.

(ii) Solve (30) with $\gamma=\gamma^{n}$. If

$$
H^{n}(\gamma)\left(\tilde{\delta}_{u}^{n}\right)^{2}<\epsilon\left|\tilde{\delta}_{u}^{n}\right|_{U}^{2},
$$

then choose $\tilde{\gamma} \in\left[0, \omega \gamma^{n}\right]$, with $0<\omega<1$ fixed, set $\gamma^{n}:=\tilde{\gamma}$ and start with (ii) again; otherwise goto (iii).

(iii) Put $\delta_{x}^{n}:=\tilde{\delta}_{x}^{n}, \hat{\lambda}^{n}:=\tilde{\lambda}^{n}$.

Remark 3.2. In the modified step (2a) the adjustment strategy for $\gamma^{n}$ reflects a compromise between $\gamma^{n}=1$, i.e. the Newton-step, and $\gamma^{n}=0$, i.e. a scaled gradient step. Of course, one may also resort to the discrete switching technique, where $\gamma^{n}=1$ is accepted if the test (33) is satisfied; otherwise $\gamma^{n}=0$ is chosen. The latter technique is numerically less expensive since one avoids an iterative adjustment of $\gamma^{n}$. Note that due to (29) and $\epsilon \in(0, c)$ the test (33) is always satisfied for $\gamma^{n}=0$. In the case of an iterative adjustment of $\gamma^{n}$, a strategy working well in practice uses the choice

$$
\tilde{\gamma}:=\min \left\{\frac{\epsilon\left|\tilde{\delta}_{u}^{n}\right|_{U}^{2}-T^{*}\left(x^{n}\right) J_{x x}\left(x^{n}\right) T\left(x^{n}\right)\left(\tilde{\delta}_{u}^{n}\right)^{2}}{T^{*}\left(x^{n}\right) e_{x x}\left(x^{n}\right) T\left(x^{n}\right)\left(\tilde{\delta}_{u}^{n}\right)^{2}}, \omega \gamma^{n}\right\}>0 .
$$

From local convergence results for SQP-methods $[4,12,15,16]$ we expect that $\gamma^{n}=1$ for iterations $n \geq N$ (for sufficiently small $\epsilon>0)$ if $\left(x^{n}, \lambda^{n}\right)$ for $n \geq N$ remains in a sufficiently small neighborhood of a local minimizer $x^{*}$ with multiplier $\lambda^{*}$. Therefore, the fast local convergence rates of SQP-methods would be preserved. A detailed analysis of these aspects is part of Section 4 .

\subsection{Line search}

We turn now towards the second issue concerning a globalization strategy for the underlying SQP-method the line search. In the course of the SQP-iteration (outer loop) a compromise between the descent of $J(x)$ and the reduction of the violation of $e(x)=0$ must be found. The way how we realize this compromise is the usage of a line search based on a suitably chosen merit functional. There exist many proposals for merit functionals in the literature; see e.g. $[3,17,19]$. Here we choose the exact penalty functional

$$
\phi\left(x+\alpha \delta_{x}\right)=J\left(x+\alpha \delta_{x}\right)+\mu\left|e\left(x+\alpha \delta_{x}\right)\right|_{Z^{*}},
$$

where $\alpha \in[0,1]$, and $\mu>0$ is a parameter penalizing violations of the constraint $e=0$. In case that $x=x^{n}$ and $\delta_{x}=\delta_{x}^{n}$ we shall use the notation $\phi^{n}(\alpha)$ instead of $\phi\left(x^{n}+\alpha \delta_{x}^{n}\right)$. 
Our favor for the exact penalty function comes from the fact that-apart from taking the norm-it introduces no additional non-linearity (and is thus computationally inexpensive and easy to analyze) and in the case of the computation of inexact solutions to the quadratic problems (19) (inner loop), in our tests it typically outperforms other classical choices. Moreover, observe that we use only one penalty parameter for both components $e^{1}$ and $e^{2}$ of $e$. This is in contrast to frequent proposals of one penalty parameter per constraint. Our choice is motivated by the linearity of $e^{2}$ and the fact that typically the starting values satisfy the initial condition $y^{0}(0)=y_{0}$. Then due to the nature of $(30) e^{2}\left(x^{n}\right)=0$ is satisfied in case of exact subproblem solutions for all $n$. Hence violations of $e^{2}$ do not occur in the course of the iteration.

In a first order sense we use the approximation

$$
\bar{\phi}\left(x+\alpha \delta_{x}\right)=J(x)+\alpha J_{x}(x) \delta_{x}+\mu\left|e(x)+\alpha e_{x}(x) \delta_{x}\right|_{Z^{*}}
$$

Again we use $\bar{\phi}^{n}(\alpha)$ if $x=x^{n}$ and $\delta_{x}=\delta_{x}^{n}$.

For appropriately chosen penalty parameter $\mu$, a suitable test whether we can accept the above mentioned compromise is

$$
\phi^{n}(\alpha)-\phi^{n}(0) \leq \beta \alpha\left(\bar{\phi}^{n}(1)-\bar{\phi}^{n}(0)\right) \quad \text { with } \quad 0<\beta<\frac{1}{2}
$$

Thus the compromise is achieved by suitably adjusting the step-size $\alpha$. In fact, if (35) is not satisfied, then a smaller value for $\alpha$ is chosen and tested again. The rule (35) is also known as Armijo rule [4,8].

We still have to clarify two facts. First we have to discuss the appropriate choice of $\mu$ which shall guarantee that the right hand side of (35) is strictly negative and vanishes only if a solution to the nonlinear first order system (13) of (5) is found. Moreover, the rule for efficiently choosing $\alpha$ has to be discussed.

Concerning the suitable choice of $\mu$ the following result is important.

Lemma 3.3. Assume that $\left(x^{n}, \hat{\lambda}^{n}\right)$ does not satisfy (13). If the penalty parameter $\mu$ satisfies

$$
\mu \geq\left|e_{y}^{-*}\left(x^{n}\right) L_{11}^{n}\left(\gamma^{n}\right) e_{y}^{-1}\left(x^{n}\right)\left(e\left(x^{n}\right)+2 e_{u}\left(x^{n}\right) \delta_{u}^{n}\right)-\hat{\lambda}^{n}\right|_{Z}+\epsilon_{1}
$$

for some fixed $\epsilon_{1}>0$, then

$$
\eta^{n}:=\bar{\phi}^{n}(1)-\bar{\phi}^{n}(0) \leq-\max \left\{\epsilon\left|\delta_{u}^{n}\right|_{U}^{2}, \epsilon_{1}\left|e\left(x^{n}\right)\right|_{Z^{*}}\right\}<0
$$

Proof. Assume that $e\left(x^{n}\right) \neq 0$. Using (30) and the structure of $L^{n}\left(\gamma^{n}\right)$ we obtain

$$
\begin{aligned}
\bar{\phi}^{n}(1)-\bar{\phi}^{n}(0)= & J_{x}\left(x^{n}\right) \delta_{x}^{n}+\mu\left|e\left(x^{n}\right)+e_{x}\left(x^{n}\right) \delta_{x}^{n}\right|_{Z^{*}}-\mu\left|e\left(x^{n}\right)\right|_{Z^{*}} \\
= & -L^{n}\left(\gamma^{n}\right)\left(\delta_{x}^{n}\right)^{2}-\left\langle\hat{\lambda}^{n}, e_{x}\left(x^{n}\right) \delta_{x}^{n}\right\rangle_{Z, Z^{*}}-\mu\left|e\left(x^{n}\right)\right|_{Z^{*}} \\
= & -\left\langle L_{11}^{n}\left(\gamma^{n}\right)\left(e_{y}^{-1}\left(x^{n}\right)\left(e\left(x^{n}\right)+e_{u}\left(x^{n}\right) \delta_{u}^{n}\right)\right), e_{y}^{-1}\left(x^{n}\right)\left(e\left(x^{n}\right)+e_{u}\left(x^{n}\right) \delta_{u}^{n}\right)\right\rangle \\
& -J_{u u}\left(x^{n}\right)\left(\delta_{u}^{n}\right)^{2}+\left\langle\hat{\lambda}^{n}, e\left(x^{n}\right)\right\rangle_{Z, Z^{*}}-\mu\left|e\left(x^{n}\right)\right|_{Z^{*}} \\
= & -\left\langle T^{*}\left(x^{n}\right) L^{n}\left(\gamma^{n}\right) T\left(x^{n}\right) \delta_{u}^{n}, \delta_{u}^{n}\right\rangle_{U}-\left\langle e_{y}^{-*}\left(x^{n}\right) L_{11}^{n}\left(\gamma^{n}\right) e_{y}^{-1}\left(x^{n}\right) e\left(x^{n}\right), e\left(x^{n}\right)\right\rangle_{Z, Z^{*}} \\
& -2\left\langle\left(e_{y}^{-*}\left(x^{n}\right) L_{11}^{n}\left(\gamma^{n}\right) e_{y}^{-1}\left(x^{n}\right) e_{u}\left(x^{n}\right) \delta_{u}^{n}, e\left(x^{n}\right)\right\rangle_{Z, Z^{*}}+\left\langle\hat{\lambda}^{n}, e\left(x^{n}\right)\right\rangle_{Z, Z^{*}}-\mu\left|e\left(x^{n}\right)\right|_{Z^{*}}\right. \\
\leq & -\left\langle T^{*}\left(x^{n}\right) L^{n}\left(\gamma^{n}\right) T\left(x^{n}\right) \delta_{u}^{n}, \delta_{u}^{n}\right\rangle_{U}+\left(\mid e_{y}^{-*}\left(x^{n}\right) L_{11}^{n}\left(\gamma^{n}\right) e_{y}^{-1}\left(x^{n}\right)\left(e\left(x^{n}\right)\right.\right. \\
& \left.\left.+2 e_{u}\left(x^{n}\right) \delta_{u}^{n}\right)-\left.\hat{\lambda}^{n}\right|_{Z}-\mu\right)\left|e\left(x^{n}\right)\right|_{Z^{*}}
\end{aligned}
$$


Since

$$
H^{n}(\gamma)\left(\delta_{u}^{n}\right)^{2} \geq \epsilon\left|\delta_{u}^{n}\right|_{U}^{2}
$$

the choice (36) yields (37).

On the other hand, if $e\left(x^{n}\right)=0$, then the assertion holds whenever $\left|\delta_{u}^{n}\right|_{U} \neq 0$. If, however, $\left|\delta_{u}^{n}\right|_{U}=0$, then from $e\left(x^{n}\right)=0$ and (25) we obtain $\delta_{y}^{n}=0$. But then $\left(x^{n}, \hat{\lambda}^{n}\right)$ satisfies the first order conditions (13) for the nonlinear problem which contradicts the assumption of the lemma.

Notice that due to our backward substitution technique (25-27) all the terms needed for computing $\mu$ are already available. Only the norm has to be evaluated. An alternative to this procedure is as follows: Check whether (37) holds. If this is not the case, then enlarge $\mu$ e.g. by a factor greater than one and test (37) again. Now one is released from computing the norm in (36), only the expected descent $\eta^{n}$ has to be computed repeatedly. The repeated computation involves solely scalar operations.

Next we turn to the rule for determining $\alpha$ in every iteration. For this purpose we throughout invoke the following assumption:

$$
J\left(x+\delta_{x}\right)=J(x)+J_{x}(x) \delta_{x}+\frac{1}{2} J_{x x}(x)\left(\delta_{x}\right)^{2} \quad \text { for } x, \delta_{x} \in X .
$$

Due to the high computational cost (like repeated evaluation of norms) we must avoid adjustment schemes based on backtracking or interpolation [6]. For our very specific problem the next lemma provides an explicit expression for a suitable step-size. The only additional cost is one evaluation of a norm.

Lemma 3.4. Assume that $\mu$ is chosen according to Lemma 3.3. Then for each $n \in \mathbb{N}$ there exists $\bar{\alpha}^{n} \in(0,1]$ such that

$$
\phi^{n}(\alpha)-\phi^{n}(0) \leq \beta \alpha \eta^{n}<0 \quad \text { for all } \alpha \in\left(0, \bar{\alpha}^{n}\right]
$$

is satisfied, with $0<\beta<\frac{1}{2}$ fixed and

$$
\bar{\alpha}^{n}=\min \left\{1,2(\beta-1) \eta^{n}\left(J_{x x}\left(x^{n}\right)\left(\delta_{x}^{n}\right)^{2}+2 \mu\left|\left(\delta_{y}^{n} \cdot \nabla\right) \delta_{y}^{n}\right|_{L^{2}\left(V^{*}\right)}\right)^{-1}\right\}
$$

Proof. First observe that

$$
\begin{aligned}
\phi^{n}(\alpha)= & J\left(x^{n}\right)+\alpha J_{x}\left(x^{n}\right) \delta_{x}^{n}+\frac{\alpha^{2}}{2} J_{x x}\left(x^{n}\right)\left(\delta_{x}^{n}\right)^{2}+\mu\left|e^{1}\left(x^{n}\right)+\alpha e_{x}^{1}\left(x^{n}\right) \delta_{x}^{n}+\alpha^{2}\left(\delta_{y}^{n} \cdot \nabla\right) \delta_{y}^{n}\right|_{L^{2}\left(V^{*}\right)} \\
& +\mu\left|e^{2}\left(x^{n}\right)+\alpha e_{x}^{2}\left(x^{n}\right) \delta_{x}^{n}\right|_{H} .
\end{aligned}
$$


Therefore, for $\alpha \in[0,1]$ we have

$$
\begin{aligned}
\phi^{n}(\alpha)-\phi^{n}(0) \leq & (1-\alpha) J\left(x^{n}\right)+\alpha J\left(x^{n}\right)-J\left(x^{n}\right)+\alpha J_{x}\left(x^{n}\right) \delta_{x}^{n} \\
& +\frac{\alpha^{2}}{2} J_{x x}\left(x^{n}\right)\left(\delta_{x}^{n}\right)^{2}+\mu\left|(1-\alpha) e^{1}\left(x^{n}\right)+\alpha e^{1}\left(x^{n}\right)+\alpha e_{x}^{1}\left(x^{n}\right) \delta_{x}^{n}\right|_{L^{2}\left(V^{*}\right)} \\
& +\mu \alpha^{2}\left|\left(\delta_{y}^{n} \cdot \nabla\right) \delta_{y}^{n}\right|_{L^{2}\left(V^{*}\right)}+\mu\left|(1-\alpha) e^{2}\left(x^{n}\right)+\alpha e^{2}\left(x^{n}\right)+\alpha e_{x}^{2}\left(x^{n}\right) \delta_{x}^{n}\right|_{H} \\
& -\mu\left(\left|e^{1}\left(x^{n}\right)\right|_{L^{2}\left(V^{*}\right)}+\left|e^{2}\left(x^{n}\right)\right|_{H}\right) \\
\leq & (1-\alpha)\left[J\left(x^{n}\right)+\mu\left|e^{1}\left(x^{n}\right)\right|_{L^{2}\left(V^{*}\right)}+\mu\left|e^{2}\left(x^{n}\right)\right|_{H}\right] \\
& +\alpha\left[J\left(x^{n}\right)+J_{x}\left(x^{n}\right) \delta_{x}^{n}+\mu\left|e^{1}\left(x^{n}\right)+e_{x}^{1}\left(x^{n}\right) \delta_{x}^{n}\right|_{L^{2}\left(V^{*}\right)}\right. \\
& \left.+\mu\left|e^{2}\left(x^{n}\right)+e_{x}^{2}\left(x^{n}\right) \delta_{x}^{n}\right|_{H}\right]-\left[J\left(x^{n}\right)+\mu\left|e^{1}\left(x^{n}\right)\right|_{L^{2}\left(V^{*}\right)}+\mu\left|e^{2}\left(x^{n}\right)\right|_{H}\right] \\
& +\frac{\alpha^{2}}{2} J_{x x}\left(x^{n}\right)\left(\delta_{x}^{n}\right)^{2}+\mu \alpha^{2}\left|\left(\delta_{y}^{n} \cdot \nabla\right) \delta_{y}^{n}\right|_{L^{2}\left(V^{*}\right)} \\
= & (1-\alpha) \bar{\phi}^{n}(0)+\alpha \bar{\phi}^{n}(1)-\bar{\phi}^{n}(0)+\alpha^{2}\left(\frac{1}{2} J_{x x}\left(x^{n}\right)\left(\delta_{x}^{n}\right)^{2}+\mu\left|\left(\delta_{y}^{n} \cdot \nabla\right) \delta_{y}^{n}\right|_{L^{2}\left(V^{*}\right)}\right) \\
= & \alpha \eta^{n}+\alpha^{2}\left(\frac{1}{2} J_{x x}\left(x^{n}\right)\left(\delta_{x}^{n}\right)^{2}+\mu\left|\left(\delta_{y}^{n} \cdot \nabla\right) \delta_{y}^{n}\right|_{L^{2}\left(V^{*}\right)}\right)
\end{aligned}
$$

Obviously, the line search test (35) is satisfied if

$$
\alpha \eta^{n}+\alpha^{2}\left(\frac{1}{2} J_{x x}\left(x^{n}\right)\left(\delta_{x}^{n}\right)^{2}+\mu\left|\left(\delta_{y}^{n} \cdot \nabla\right) \delta_{y}^{n}\right|_{L^{2}\left(V^{*}\right)}\right) \leq \beta \alpha \eta^{n} .
$$

Some simple manipulations show that the above inequality is fulfilled for

$$
\alpha \leq 2(\beta-1) \eta^{n}\left(J_{x x}\left(x^{n}\right)\left(\delta_{x}^{n}\right)^{2}+2 \mu\left|\left(\delta_{y}^{n} \cdot \nabla\right) \delta_{y}^{n}\right|_{L^{2}\left(V^{*}\right)}\right)^{-1}=: \tilde{\alpha}^{n}
$$

Then the assertion follows for $\bar{\alpha}^{n}:=\min \left\{1, \tilde{\alpha}^{n}\right\}$.

Lemma 3.4 is used to replace step $(2 \mathrm{~b})$ of the SQP-algorithm by:

\section{Step (2b)}

Compute $\mu$ according to Lemma 3.3, and put $\mu^{n}=\max \left\{\mu^{n-1}, \mu\right\}$. Compute $\alpha^{n}:=\bar{\alpha}^{n}$ according to (38), with $\mu$ replaced by $\mu^{n}$, and update $\left(x^{n+1}, \lambda^{n+1}\right)=\left(x^{n}, \lambda^{n}\right)+\alpha^{n}\left(\delta_{x}^{n}, \delta_{\lambda}^{n}\right)$, with $\delta_{\lambda}^{n}=\hat{\lambda}^{n}-\lambda^{n}$. Set $n=n+1$.

Finally, observe that from (40) in the proof of Lemma 3.4 it follows that

$$
\mu^{n} \alpha^{n}\left|e_{x x}\left(x^{n}\right)\left(\delta_{x}^{n}\right)^{2}\right|_{Z^{*}} \leq 2(\beta-1) \eta^{n}
$$

\section{Convergence analysis}

In this section we are concerned with global as well as local convergence analysis of the globalized SQPmethod. For convenience we recall the overall globalized SQP-algorithm.

\section{Algorithm 4.1. Globalized SQP-algorithm}

1. Choose $\left(x^{0},(\lambda)^{0}\right) \in X \times Z, \epsilon, \epsilon_{1} \in \mathbb{R}^{+}$sufficiently small, and $\mu^{0} \in \mathbb{R}^{+}$. Set $n=0$.

2. Do until convergence
(a) (i) Set $\gamma^{n}:=1$. 
(ii) Solve (30) with $\gamma=\gamma^{n}$. If

$$
H^{n}\left(\gamma^{n}\right)\left(\tilde{\delta}_{u}^{n}\right)^{2}<\epsilon\left|\tilde{\delta}_{u}^{n}\right|_{U}^{2}
$$

then choose $\tilde{\gamma} \in\left[0, \omega \gamma^{n}\right]$, with $0<\omega<1$ fixed, set $\gamma^{n}:=\tilde{\gamma}$ and start with (ii) again; otherwise goto (iii).

(iii) Put $\delta_{x}^{n}:=\tilde{\delta}_{x}^{n}, \hat{\lambda}^{n}:=\tilde{\lambda}^{n}$.

(b) Compute $\mu$ according to Lemma 3.3, and put $\mu^{n}=\max \left\{\mu^{n-1}, \mu\right\}$. Compute $\alpha^{n}:=\bar{\alpha}^{n}$ according to (38), with $\mu$ replaced by $\mu^{n}$, and update $\left(x^{n+1}, \lambda^{n+1}\right)=\left(x^{n}, \lambda^{n}\right)+\alpha^{n}\left(\delta_{x}^{n}, \delta_{\lambda}^{n}\right)$, with $\delta_{\lambda}^{n}=\hat{\lambda}^{n}-\lambda^{n}$. Set $n=n+1$.

\subsection{Global convergence}

Now we prove the main global convergence result which states that for arbitrary starting points the accumulation points of $\left\{\left(x^{n}, \lambda^{n}\right)\right\}_{n \in \mathbb{N}}$ satisfy the (nonlinear) first order conditions (13).

Theorem 4.2. Suppose there exist (uniform) constants $M_{1}, M_{2}, M_{3}, \bar{\mu} \in \mathbb{R}^{+}$and an iteration index $N$ such that

(i) $\max \left\{\left|x^{n}\right|_{X},\left|\delta_{x}^{n}\right|_{X},\left|\lambda^{n}\right|_{Z}\right\} \leq M_{1}$;

(ii) $J_{x x}\left(x^{n}\right)\left(\delta_{x}^{n}\right)^{2} \leq M_{2}$ and $\left.\mid\left(\delta_{y}^{n} \cdot \nabla\right) \delta_{y}^{n}\right)\left.\right|_{L^{2}\left(V^{*}\right)} \leq M_{3}$;

(iii) $\mu^{n}=\bar{\mu}$ for all $n \geq N$.

Then

1. $\lim _{n \rightarrow \infty}\left|\delta_{u}^{n}\right|_{U}=0$ and $\lim _{n \rightarrow \infty}\left|\delta_{y}^{n}\right|_{W}=0$;

2. every accumulation point $(\bar{x}, \bar{\lambda}) \in X \times Z$ satisfies

$$
\begin{aligned}
e(\bar{x}) & =0, \\
J_{x}(\bar{x})+e_{x}^{*}(\bar{x}) \bar{\lambda} & =0 .
\end{aligned}
$$

Proof. First we prove that $\lim _{n \rightarrow \infty} \eta^{n}=0$. For this purpose, assume that there exists an infinite number of iterations $(n(l))_{l \in \mathbb{N}}$ with $\eta^{n(l)} \leq \hat{\eta}$ for arbitrarily fixed $\hat{\eta} \in\left(\frac{1}{2}(\beta-1)^{-1}\left(M_{2}+\bar{\mu} M_{3}\right), 0\right)$. Then from our assumptions and Lemma 3.4 we deduce for sufficiently large $l$

$$
\bar{\alpha}^{n(l)} \geq 2(\beta-1) \hat{\eta}\left(M_{2}+2 \bar{\mu} M_{3}\right)^{-1}>0 .
$$

The line search test (35) yields

$$
\phi^{n(l)}\left(\alpha^{n(l)}\right)-\phi^{n(l)}(0) \leq \beta \alpha^{n(l)} \eta^{n(l)} \leq 2 \beta(\beta-1) \hat{\eta}^{2}\left(M_{2}+2 \bar{\mu} M_{3}\right)^{-1}<0
$$

for all sufficiently large $l$. Note further that for $l$ large enough $\phi^{n(l)+1}(0)=\phi^{n(l)}\left(\alpha^{n(l)}\right)$, which is due to $\mu^{n(l)}=\bar{\mu}$ for $n(l) \geq N$. Hence, $\left\{\phi^{n(l)}(0)\right\}_{n(l) \geq N}$ is strictly decreasing and uniformly bounded from below by assumption. This implies

$$
\lim _{l \rightarrow \infty}\left(\phi^{n(l)}\left(\alpha^{n(l)}\right)-\phi^{n(l)}(0)\right)=0 .
$$

This contradicts (45). Thus we have

$$
\lim _{n \rightarrow \infty} \eta^{n}=0
$$


From (37) and the proof of Lemma 3.3 it immediately follows that

$$
\lim _{n \rightarrow \infty}\left|e\left(x^{n}\right)\right|_{Z^{*}}=0, \quad \lim _{n \rightarrow \infty}\left|\delta_{u}^{n}\right|_{U}=0, \quad \text { and } \quad \lim _{n \rightarrow \infty} T^{*}\left(x^{n}\right) L^{n}\left(\gamma^{n}\right) T\left(x^{n}\right)\left(\delta_{u}^{n}\right)^{2}=0 .
$$

This proves (42) and the first part of assertion 1. The system (30) yields

$$
\left|e_{y}\left(x^{n}\right) \delta_{y}^{n}\right|_{Z^{*}} \leq\left|e\left(x^{n}\right)\right|_{Z^{*}}+\left|B \delta_{u}^{n}\right|_{L^{2}\left(V^{*}\right)}
$$

From the invertibility of $e_{y}$, the boundedness of $\left(x^{n}\right)_{n \in \mathbb{N}}$, and (47) it follows that

$$
\lim _{n \rightarrow \infty}\left|\delta_{y}^{n}\right|_{W}=0
$$

This proves the second part of assertion 1. From (31) we obtain

$$
\left|J_{y}\left(x^{n}\right)+e_{y}^{*}\left(x^{n}\right) \hat{\lambda}^{n}\right|_{W^{*}} \leq\left|L_{11}^{n}\left(\gamma^{n}\right) \delta_{y}^{n}\right|_{W^{*}} .
$$

Above the right hand side vanishes due to (48), and the boundedness of $\left|L_{11}^{n}\left(\gamma^{n}\right)\right|_{L^{2}\left(V^{*}\right) \leftarrow L^{2}(V)}$ independently of $n$ by assumption. From (32) and using (31) we derive

$$
\left|J_{u}\left(x^{n}\right)+e_{u}^{*}\left(x^{n}\right) \hat{\lambda}^{n}\right|_{U} \leq\left|H^{n}\left(\gamma^{n}\right)\right|_{\mathcal{L}(U)}\left|\delta_{u}^{n}\right|_{U}+\left|e_{u}\left(x^{n}\right)^{*} e_{y}^{-*}\left(x^{n}\right) L_{11}^{n}\left(\gamma^{n}\right) e_{y}^{-1}\left(x^{n}\right) e_{u}\left(x^{n}\right)\right|_{\mathcal{L}(U)}\left|\delta_{u}^{n}\right|_{U} .
$$

Note that due to our assumptions the factors in front of $\left|\delta_{u}^{n}\right|$ above are uniformly bounded. Hence, assertion (43) then follows from $(47-50)$.

\subsection{Local convergence}

A fast local rate of convergence for the globalized SQP-method is related to the acceptance of both $\gamma^{n}=1$ and $\alpha^{n}=1$ for all $n \geq N$ with $N \in \mathbb{N}$ sufficiently large. Then the method reduces to a well-known SQP-algorithm and thus the local convergence results of [12] apply.

Let us start by proving that $\gamma^{n}=1$ at sufficiently large iteration levels $n$.

Lemma 4.3. Assume that $\lim _{n \rightarrow \infty}\left|\left(x^{n}, \lambda^{n}\right)-\left(x^{*}, \lambda^{*}\right)\right|_{X \times Z}=0$ with $\left(x^{*}, \lambda^{*}\right) \in X \times Z$ satisfying the strong second order sufficient conditions (16), and $\epsilon>0$ is sufficiently small (see (33)). Then there exists $N_{\gamma} \in \mathbb{N}$ such that $\gamma^{n}=1$ for all $n \geq N_{\gamma}$.

Proof. From [12] (Th. 5.8), we deduce that under the assumptions of the lemma there exist a neighborhood $U\left(x^{*}\right) \times V\left(\lambda^{*}\right) \subset X \times Z$ and $\underline{\epsilon}>0$ such that for all $(x, \lambda) \in U\left(x^{*}\right) \times V\left(\lambda^{*}\right)$

$$
T^{*}(x) L_{x x}(x, \lambda) T(x)\left(\delta_{u}\right)^{2} \geq \underline{\epsilon}\left|\delta_{u}\right|_{U}^{2}>0
$$

for $\delta_{u} \in U, \delta_{u} \neq 0$. Since $\left(x^{n}, \lambda^{n}\right) \rightarrow\left(x^{*}, \lambda^{*}\right)$ in $X \times Z$, there exists $N_{\gamma} \in \mathbb{N}$ such that $\left(x^{n}, \lambda^{n}\right) \in U\left(x^{*}\right) \times V\left(\lambda^{*}\right)$ for all $n \geq N_{\gamma}$. Now choose $0<\epsilon \leq \underline{\epsilon}$, then the assertion follows.

In the remainder of this section we assume that

$$
\lim _{n \rightarrow \infty}\left|\left(x^{n}, \lambda^{n}\right)-\left(x^{*}, \lambda^{*}\right)\right|_{X \times Z}=0
$$

with $\left(x^{*}, \lambda^{*}\right)$ like in Lemma 4.3. From local convergence results for SQP-methods $[4,12,15,16]$ it is known that no line search is needed for obtaining a convergent algorithm. In our case this can be seen from [12] (Th. 5.7) 
and the fact that the iteration in step (2a) of the algorithm is equivalent to applying Newton's method to the first order system

$$
\begin{aligned}
L_{x}(x, \lambda) & =0, \\
e(x) & =0 .
\end{aligned}
$$

We further deduce the existence of a neighborhood $U^{*} \times V^{*} \subset X \times Z$ of $\left(x^{*}, \lambda^{*}\right)$ such that $\left(x^{n}+\delta_{x}^{n}, \hat{\lambda}^{n}\right) \in$ $U^{*} \times V^{*}$ for all $n \geq 1$ provided that $\left(x^{0},(\lambda)^{0}\right) \in U^{*} \times V^{*}$. Since $x^{n+1}=x^{n}+\alpha^{n} \delta_{x}^{n}, \lambda^{n+1}=\lambda^{n}+\alpha^{n} \delta_{\lambda}^{n}$, and $\alpha^{n} \in(0,1]$ by our line search, our globalized SQP-method is locally convergent. Thus, Lemma 4.3 ensures that the system (18) is retained for large iteration levels. However, our favor for the exact penalty functional $\phi$ (see (34)) may prevent $\alpha^{n}=1$ for sufficiently large $n$. Hence, the fast local convergence behavior of Newton's method [12] may be impeded by our line search. The reason for this comes from the fact that $e$ occurs only in its linearized form in (18), respectively (19). As a consequence the quantity

$$
v(x)=\mu^{n}|e(x)|_{Z^{*}}-\left\langle\lambda^{*}, e(x)\right\rangle_{Z, Z^{*}}
$$

is only of order $\mathcal{O}\left(\left|\delta_{x}^{n}\right|_{X}^{2}\right)$ instead of $\mathcal{O}\left(\left|\delta_{x}^{n}\right|_{X}^{2}\right)$ at $x=x^{n}+\delta_{x}^{n}$. Here $\mathcal{O}$ and $\mathcal{O}$ denote the Landau symbols. To see the importance of the fact that

$$
v\left(x^{n}+\delta_{x}^{n}\right)=\mathcal{O}\left(\left|\delta_{x}^{n}\right|_{X}^{2}\right)
$$

first assume that the sequence $\left(x^{n}\right)$ satisfies

$$
\left|x^{n}+\delta_{x}^{n}-x^{*}\right|_{X}=\mathcal{O}\left(\left|x^{n}-x^{*}\right|_{X}\right) .
$$

In this case, we obviously have $\left|\delta_{x}^{n}\right|_{X}=\mathcal{O}\left(\left|x^{n}-x^{*}\right|_{X}\right)$. Next, notice that for $\bar{\mu} \geq\left|\lambda^{*}\right|_{Z}$

$$
\begin{aligned}
L\left(x^{n}+\delta_{x}^{n}, \lambda^{*}\right) & =L\left(x^{*}, \lambda^{*}\right)+\frac{1}{2} L_{x x}\left(x^{*}, \lambda^{*}\right)\left(x^{n}+\delta_{x}^{n}-x^{*}\right)^{2}, \\
\phi\left(x^{n}\right) & \geq L\left(x^{n}, \lambda^{*}\right) \geq L\left(x^{*}, \lambda^{*}\right)+\tau\left|\delta_{x}^{n}\right|_{X}^{2},
\end{aligned}
$$

for some sufficiently small $\tau>0$, where (51) is due to a Taylor expansion and $L_{x}\left(x^{*}, \lambda^{*}\right)=0$, and (52) is deduced from the strong second order sufficient conditions (16) for large $n$. Hence,

$$
\begin{aligned}
\phi\left(x^{n}+\delta_{x}^{n}\right)= & L\left(x^{n}+\delta_{x}^{n}, \lambda^{*}\right)+v\left(x^{n}+\delta_{x}^{n}\right) \\
\leq & L\left(x^{n}, \lambda^{*}\right)-\tau\left|\delta_{x}^{n}\right|_{X}^{2}+\frac{1}{2} L_{x x}\left(x^{*}, \lambda^{*}\right)\left(x^{n}+\delta_{x}^{n}-x^{*}\right)^{2} \\
& +v\left(x^{n}+\delta_{x}^{n}\right) .
\end{aligned}
$$

We continue estimation in (53) and obtain

$$
\phi\left(x^{n}+\delta_{x}^{n}\right) \leq \phi\left(x^{n}\right)-\tau\left|\delta_{x}^{n}\right|_{X}^{2}+\mathcal{O}\left(\left|\delta_{x}^{n}\right|_{X}^{2}\right)+v\left(x^{n}+\delta_{x}^{n}\right)
$$

Since $\left|\delta_{x}^{n}\right|_{X} \rightarrow 0$ by Theorem 4.2 , and if

$$
v\left(x^{n}+\delta_{x}^{n}\right)=\mathcal{O}\left(\left|\delta_{x}^{n}\right|_{X}^{2}\right)
$$

then (54) guarantees that for sufficiently large $n$ and appropriate choices of $\beta$ and $\bar{\mu}$ the line search test (35) is satisfied for $\alpha^{n}=1$. Hence, the local convergence results for Newton's method apply [18]. 
In order to establish the desired relation (55) we consider the following modification of the search direction $\delta_{x}^{n}$ : compute $\delta_{x}^{n}$ from $(30)$, and then determine

$$
\hat{\delta}_{x}^{n}:=\left(\hat{\delta}_{y}^{n}, 0\right), \text { with } \hat{\delta}_{y}^{n}:=-\frac{1}{2} e_{y}^{-1}\left(x^{n}\right) e_{y y}\left(x^{n}\right)\left(\delta_{x}^{n}\right)^{2} .
$$

The next iterate is $x^{n+1}=x^{n}+\delta_{x}^{n}+\hat{\delta}_{x}^{n}$. This approach is justified by:

Lemma 4.4. Suppose that $\hat{\delta}_{x}^{n}$ is computed by (56), and $x^{n+1}=x^{n}+\delta_{x}^{n}+\hat{\delta}_{x}^{n}$. Then $v\left(x^{n+1}\right)=\mathcal{O}\left(\left|\delta_{x}^{n}\right|_{X}^{3}\right)$.

Proof. Consider

$$
\begin{aligned}
e\left(x^{n+1}\right) & =e\left(x^{n}\right)+e_{x}\left(x^{n}\right)\left(\delta_{x}^{n}+\hat{\delta}_{x}^{n}\right)+\frac{1}{2} e_{x x}\left(x^{n}\right)\left(\delta_{x}^{n}+\hat{\delta}_{x}^{n}\right)^{2} \\
& =e_{x x}\left(x^{n}\right) \delta_{x}^{n} \hat{\delta}_{x}^{n}+\frac{1}{2} e_{x x}\left(x^{n}\right)\left(\hat{\delta}_{x}^{n}\right)^{2}
\end{aligned}
$$

where we used $e\left(x^{n}\right)+e_{x}\left(x^{n}\right) \delta_{x}^{n}=0$ by (30), and (56). Note further that

$$
e_{x x}\left(x^{n}\right) \delta_{x}^{n} \hat{\delta}_{x}^{n}+\frac{1}{2} e_{x x}\left(x^{n}\right)\left(\hat{\delta}_{x}^{n}\right)^{2}=e_{y y}\left(x^{n}\right) \delta_{y}^{n} \hat{\delta}_{y}^{n}+\frac{1}{2} e_{y y}\left(x^{n}\right)\left(\hat{\delta}_{y}^{n}\right)^{2}
$$

Exploiting the previous results, we obtain

$$
v\left(x^{n+1}\right) \leq\left(\left|\lambda^{*}\right|_{Z}+\bar{\mu}\right)\left(C_{1}\left|\delta_{y}^{n}\right|_{W}^{3}+C_{2}\left|\delta_{y}^{n}\right|_{W}^{4}\right),
$$

for some positive constants $C_{1}, C_{2}$. Here we employed the facts that $e_{x x}$ is independent of $x$, and by the boundedness of $e_{y}^{-1}$ there exists a constant $C$ independent of $n$ such that

$$
\left|\hat{\delta}_{x}^{n}\right|_{X} \leq C\left|\delta_{x}^{n}\right|_{X}^{2}
$$

This completes the proof.

One way to implement the modification of the search direction is to compute $\hat{\delta}_{x}^{n}$ as described above and perform the line search test (35) with respect to $x^{n}+\alpha \delta_{x}^{n}+\alpha^{2} \hat{\delta}_{x}^{n}$. But then the computations for obtaining an explicit expression for the analogue of $\bar{\alpha}^{n}$ (see Lem. 3.4) are more involved.

In our numerical tests we employed the following strategy: if we observe for a certain number of successive iterations that $\alpha^{n}$ stagnates at a value less than 1 , then $\hat{\delta}_{x}^{n}$ is computed. The new estimate $x^{n+1}=x^{n}+\delta_{x}^{n}+\hat{\delta}_{x}^{n}$ is accepted if (35) is satisfied for the modified direction with $\alpha=1$. Otherwise only the original search direction $\delta_{x}^{n}$ is used for the line search. Hence, the step-size choice (38) can be used.

\section{NUMERICAL VALIDATION}

The control problem considered here is of tracking type and is given by (5) with cost functional

$$
J(y, u):=\frac{1}{2} \int_{Q}|y-z|^{2} \mathrm{~d} x \mathrm{~d} t+\frac{c}{2} \int_{Q}|u|^{2} \mathrm{~d} x \mathrm{~d} t
$$

and control space $U:=L^{2}(Q)^{2}$, with $B$ denoting the injection from $U$ into $L^{2}\left(V^{*}\right)$. The first term in the cost functional values the control gain which here is to track the state $z$, and the second term measures the control cost, where $c>0$ denotes a weighting factor. 
The initial value of the uncontrolled flow is chosen as

$$
y(0, x)=e\left[\begin{array}{c}
\left(\cos 2 \pi x_{1}-1\right) \sin 2 \pi x_{2} \\
-\left(\cos 2 \pi x_{2}-1\right) \sin 2 \pi x_{1}
\end{array}\right]
$$

with $e$ denoting the Euler number, and the desired state is time dependent and given by

$$
z(t, x)=\left[\begin{array}{c}
\varphi_{x_{2}}\left(t, x_{1}, x_{2}\right) \\
-\varphi_{x_{1}}\left(t, x_{1}, x_{2}\right)
\end{array}\right]
$$

where $\varphi$ is defined through the stream function

$$
\varphi\left(t, x_{1}, x_{2}\right)=\theta\left(t, x_{1}\right) \theta\left(t, x_{2}\right)
$$

with

$$
\theta(t, s)=(1-s)^{2}(1-\cos 2 k \pi t), \quad s \in[0,1]
$$

For the results presented $c=1$.e-2, 1.e-3, $k=1$, and the time interval is chosen as $[0,1]$, i.e. T=1. For the discretization in time an equidistant grid with width $\delta t=0.01625$ is used, for the spatial discretization the Taylor-Hood finite element [13] is used on a grid containing 1024 triangles with 2113 velocity and 545 pressure nodes. The number of unknowns in the discretized control problem therefore has the magnitude $1.65 \times 10^{6}$, including the primal, adjoint and control variables.

All computations were performed on an ORIGIN $200^{T M}$. The computer code uses parts of the Navier-Stokes solver developed by Bänsch in [2].

In Figure 1 the desired flow at $T=1$ is shown. In Figure 3 the evolution of the cost functional as a function of time is documented, in Figure 2 the controlled flow together with the control action at $T=1$ for $c=1$.e-2 and $c=1 . \mathrm{e}-3$ are presented.

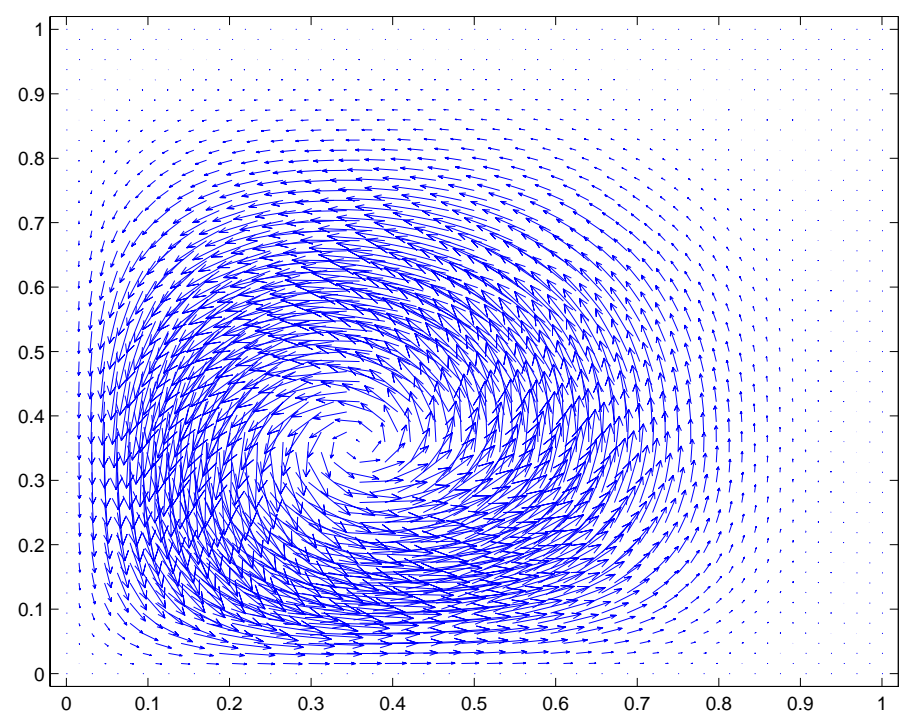

Figure 1. Desired flow at $T=1$.

Tables 1 and 2 document the performance of the globalized SQP-algorithm 4.1 for the penalties $c=1 . e-2$ and $c=1$.e-3, respectively. In both cases the algorithm was initialized with $(y, \lambda, u)^{t}=(0,0,0)^{t}$ and $\epsilon$ was 

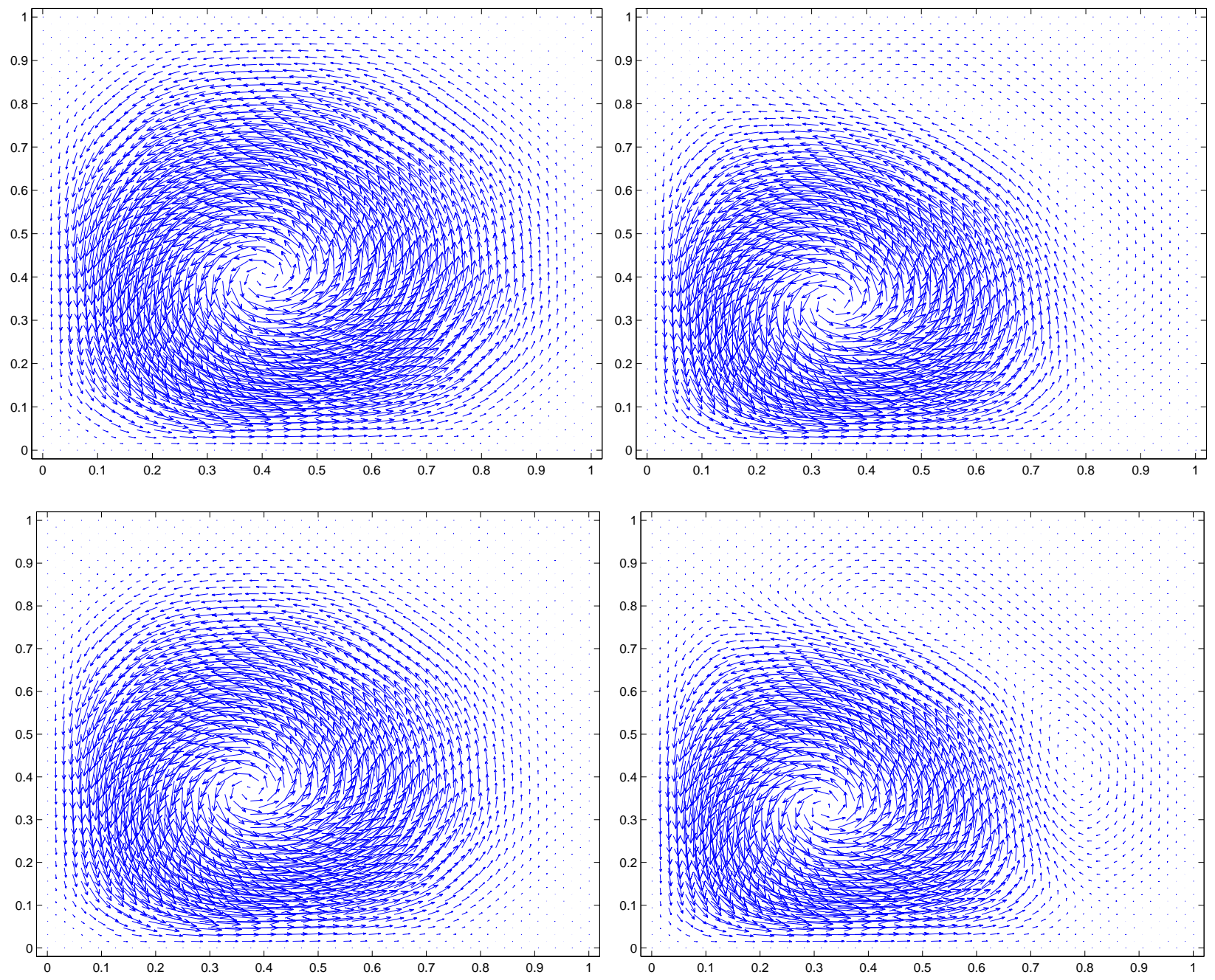

FiguRE 2. Top left, $c=1$.e-2: controlled flow; top right: control force; bottom left, $c=1$.e-3: controlled flow; bottom right: control force.

chosen as $c / 2$. To anticipate parts of the discussion we note that $\gamma^{n}=1$ and $\mu^{n}=\mu^{0}$ holds for all iterations and both values for $c$. Our experience with the $\gamma$-strategy in the context of optimal control of semi-linear partial differential equations clearly justifies the introduction of $\gamma$; see [10]. There, typically $\gamma^{n}<1$ at the starting iterations, and without the modification positive definiteness was lost.

The estimated step sizes $\alpha^{n}$ increase monotonically and the full SQP-step is accepted at $n=9$ for $c=1$.e-2 and at $n=11$ for $c=1$.e-3, respectively. Furthermore, super-linear convergence is attained with the full SQP step for both penalties.

The termination criterion for the outer iteration is chosen as $\frac{\left|L^{\prime}\left(x^{n}, \lambda^{n}\right)\right|}{L^{\prime}\left(x^{0}, \lambda^{0}\right) \mid} \leq 10^{-3}$. For the iterative solution of (2a) in Algorithm 4.1 we utilize the conjugate gradient algorithm whose termination criterion for the $j$-th iterate $\delta u_{j}^{n}$ is chosen as

$$
\frac{\left|H^{n}\left(\gamma^{n}\right) \delta u_{j}^{n}-r\left(x^{n}, \lambda^{n}\right)\right|}{\left|L^{\prime}\left(x^{0}, \lambda^{0}\right)\right|} \leq \min \left\{\left(\frac{\left|L^{\prime}\left(x^{n}, \lambda^{n}\right)\right|}{\left|L^{\prime}\left(x^{0}, \lambda^{0}\right)\right|}\right)^{\frac{3}{2}}, 10^{-2} \frac{\left|L^{\prime}\left(x^{n}, \lambda^{n}\right)\right|}{\left|L^{\prime}\left(x^{0}, \lambda^{0}\right)\right|}\right\},
$$




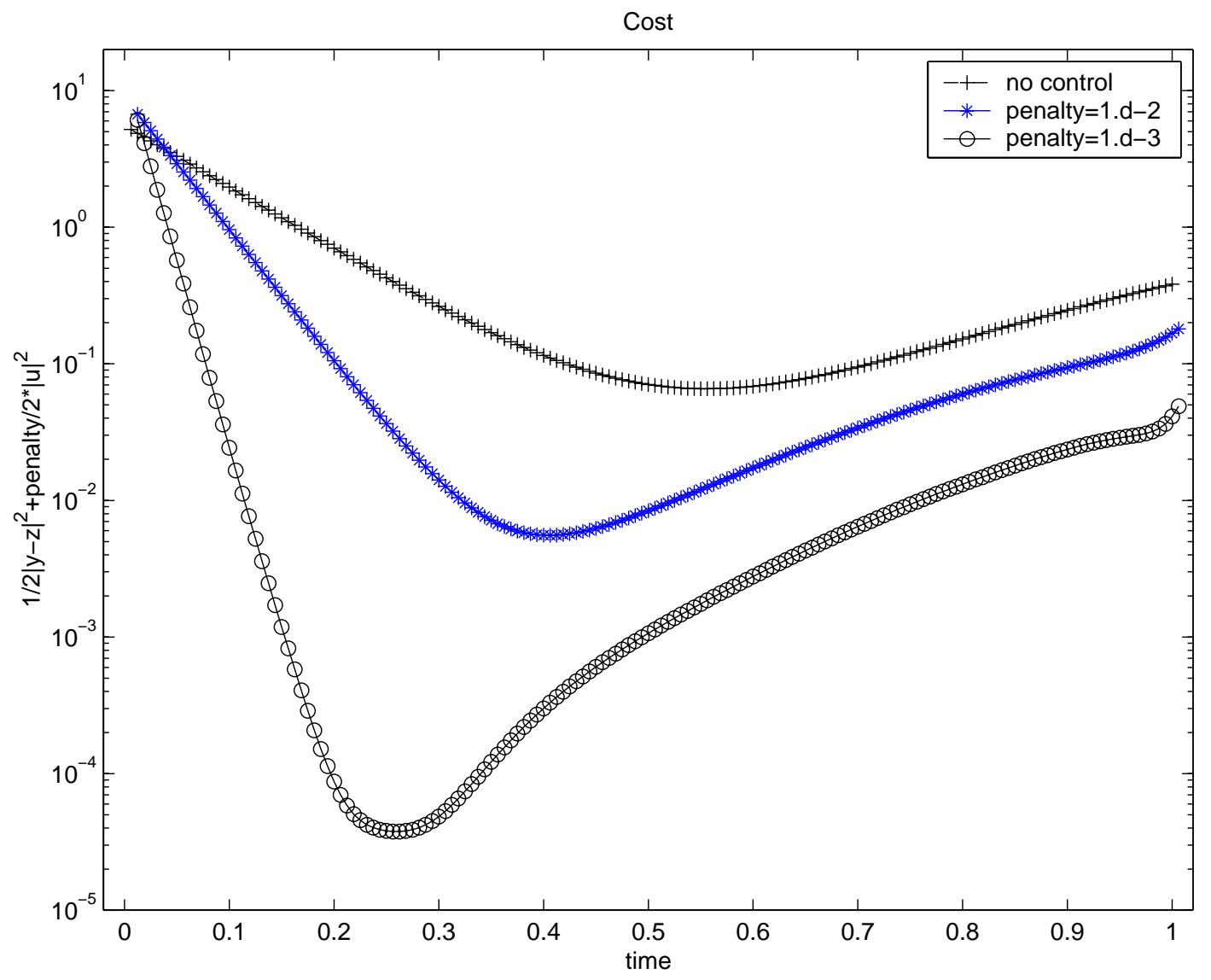

Figure 3. Evolution of cost functional.

where $r\left(x^{n}, \lambda^{n}\right)$ denotes the right-hand-side in (32). This termination criterion is motivated by stopping rules that are utilized for inexact Newton methods, say, in the finite dimensional setting in order to guarantee superlinear convergence, compare [14].

TABle 1. Performance of Algorithm 4.1 for $c=1$.e-2.

\begin{tabular}{|l|l|l|l|l|}
\hline Iteration & CG-steps & $\left|L^{\prime}\left(x^{n}, \lambda^{n}\right)\right|$ & $\left|\left(\delta_{x}^{n}, \delta_{\lambda}^{n}\right)\right|_{L^{2}(Q)}$ & $\alpha^{n}$ \\
\hline \hline 1 & 5 & $3.43 \mathrm{e}-1$ & $4.42 \mathrm{e} 0$ & $1.4796 \mathrm{e}-1$ \\
2 & 5 & $3.16 \mathrm{e}-1$ & $3.76 \mathrm{e} 0$ & $1.4910 \mathrm{e}-1$ \\
3 & 5 & $2.92 \mathrm{e}-1$ & $3.20 \mathrm{e} 0$ & $1.5046 \mathrm{e}-1$ \\
4 & 5 & $2.69 \mathrm{e}-1$ & $2.72 \mathrm{e} 0$ & $1.5225 \mathrm{e}-1$ \\
5 & 5 & $2.48 \mathrm{e}-1$ & $2.31 \mathrm{e} 0$ & $1.5461 \mathrm{e}-1$ \\
6 & 5 & $2.28 \mathrm{e}-1$ & $1.95 \mathrm{e} 0$ & $2.3557 \mathrm{e}-1$ \\
7 & 5 & $1.99 \mathrm{e}-1$ & $1.49 \mathrm{e} 0$ & $4.1146 \mathrm{e}-1$ \\
8 & 5 & $1.53 \mathrm{e}-1$ & $8.77 \mathrm{e}-1$ & $9.4451 \mathrm{e}-1$ \\
9 & 6 & $3.82 \mathrm{e}-2$ & $5.77 \mathrm{e}-2$ & $1 . \mathrm{e} 0$ \\
10 & 8 & $6.04 \mathrm{e}-3$ & $2.74 \mathrm{e}-3$ & $1 . \mathrm{e} 0$ \\
11 & 26 & $1.94 \mathrm{e}-3$ & $3.09 \mathrm{e}-4$ & $1 . \mathrm{e} 0$ \\
\hline
\end{tabular}


TABle 2. Performance of Algorithm 4.1 for $c=1 . e-3$.

\begin{tabular}{|l|l|l|l|l|}
\hline Iteration & CG-steps & $\left|L^{\prime}\left(x^{n}, \lambda^{n}\right)\right|$ & $\left|\left(\delta_{x}^{n}, \delta_{\lambda}^{n}\right)\right|_{L^{2}(Q)}$ & $\alpha^{n}$ \\
\hline \hline 1 & 11 & $3.43 \mathrm{e}-1$ & $9.82 \mathrm{e} 0$ & $1.1635 \mathrm{e}-1$ \\
2 & 11 & $3.22 \mathrm{e}-1$ & $8.67 \mathrm{e} 0$ & $1.1759 \mathrm{e}-1$ \\
3 & 11 & $3.03 \mathrm{e}-1$ & $7.64 \mathrm{e} 0$ & $1.1902 \mathrm{e}-1$ \\
4 & 11 & $2.84 \mathrm{e}-1$ & $6.73 \mathrm{e} 0$ & $1.2072 \mathrm{e}-1$ \\
5 & 11 & $2.66 \mathrm{e}-1$ & $5.91 \mathrm{e} 0$ & $1.2275 \mathrm{e}-1$ \\
6 & 11 & $2.49 \mathrm{e}-1$ & $5.18 \mathrm{e} 0$ & $1.2776 \mathrm{e}-1$ \\
7 & 11 & $2.33 \mathrm{e}-1$ & $4.51 \mathrm{e} 0$ & $1.6926 \mathrm{e}-1$ \\
8 & 11 & $2.12 \mathrm{e}-1$ & $3.74 \mathrm{e} 0$ & $2.3594 \mathrm{e}-1$ \\
9 & 11 & $1.85 \mathrm{e}-1$ & $2.85 \mathrm{e} 0$ & $3.5854 \mathrm{e}-1$ \\
10 & 11 & $1.48 \mathrm{e}-1$ & $1.82 \mathrm{e} 0$ & $6.5346 \mathrm{e}-1$ \\
11 & 11 & $8.73 \mathrm{e}-2$ & $6.26 \mathrm{e}-1$ & $1 . \mathrm{e} 0$ \\
12 & 14 & $9.69 \mathrm{e}-3$ & $2.23 \mathrm{e}-2$ & $1 . \mathrm{e} 0$ \\
13 & 15 & $2.05 \mathrm{e}-3$ & $1.70 \mathrm{e}-3$ & $1 . \mathrm{e} 0$ \\
14 & 2 & $1.00 \mathrm{e}-3$ & $1.12 \mathrm{e}-4$ & $1 . \mathrm{e} 0$ \\
\hline
\end{tabular}

\section{REFERENCES}

[1] F. Abergel and R. Temam, On some Control Problems in Fluid Mechanics. Theoret. Comput. Fluid Dyn. 1 (1990) 303-325.

[2] E. Bänsch, An adaptive Finite-Element-Strategy for the three-dimensional time-dependent Navier-Stokes Equations. J. Comput. Math. 36 (1991) 3-28.

[3] D. Bertsekas, Nonlinear Programming. Athena Scientific, Belmont, Massachusetts (1995).

[4] J.F. Bonnans et al., Optimisation Numérique. Math. Appl. 27, Springer-Verlag, Berlin (1997).

[5] O. Ghattas and J.J. Bark, Optimal control of two-and three-dimensional incompressible Navier-Stokes Flows. J. Comput. Physics 136 (1997) 231-244.

[6] P.E. Gill et al., Practical Optimization. Academic Press, San Diego, California (1981).

[7] R. Glowinski, Finite element methods for the numerical simulation of incompressible viscous flow. Introduction to the Control of the Navier-Stokes Equations. Lect. Appl. Math. 28 (1991).

[8] W.A. Gruver and E. Sachs, Algorithmic Methods in Optimal Control. Res. Notes Math. 47, Pitman, London (1980).

[9] M. Heinkenschloss, Formulation and analysis of a sequential quadratic programming method for the optimal Dirichlet boundary control of Navier-Stokes flow, in Optimal Control: Theory, Algorithms, and Applications, Kluwer Academic Publishers B.V. (1998) 178-203.

[10] M. Hintermüller, On a globalized augmented Lagrangian-SQP algorithm for nonlinear optimal control problems with box constraints, in Fast solution methods for discretized optimization problems, K.-H. Hoffmann, R.H.W. Hoppe and V. Schulz Eds., Internat. Ser. Numer. Math. 138 (2001) 139-153.

[11] M. Hinze, Optimal and instantaneous control of the instationary Navier-Stokes equations, Habilitationsschrift (1999). Fachbereich Mathematik, Technische Universität Berlin, download see http://www.math.tu-dresden.de/ hinze/publications.html.

[12] M. Hinze and K. Kunisch, Second order methods for optimal control of time-dependent fluid flow. SIAM J. Optim. Control 40 (2001) 925-946.

[13] P. Hood and C. Taylor, A numerical solution of the Navier-Stokes equations using the finite element technique. Comput. E Fluids 1 (1973) 73-100.

[14] C.T. Kelley, Iterative Methods for Linear and Nonlinear Equations. SIAM (1995).

[15] F.S. Kupfer, An infinite-dimensional convergence theory for reduced SQP-methods in Hilbert space. SIAM J. Optim. 6 (1996).

[16] E. Polak, Optimization. Appl. Math. Sci. 124, Springer-Verlag, New York (1997). 
[17] M.J.D. Powell, Variable metric methods for constrained optimization, in Mathematical Programming, The State of the Art, Eds. Bachem, Grötschel, Korte, Bonn (1982).

[18] W.C. Rheinboldt, Methods for Solving Systems of Nonlinear Equations. CBMS-NSF Regional Conference Series in Applied Mathematics 70, SIAM, Philadelphia (1998).

[19] K. Schittkowski, On the convergence of a sequential quadratic programming method with an augmented Lagrangian line search function. Math. Operationsforschung u. Statist, Ser. Optim. 14 (1983) 197-216.

[20] R. Temam, Navier-Stokes Equations. North-Holland (1979).

To access this journal online:

www.edpsciences.org 